\title{
SPATIAL HETEROGENEITY OF THE TUCURUÍ RESERVOIR (STATE OF PARÁ, AMAZONIA, BRAZIL) AND THE DISTRIBUTION OF ZOOPLANKTONIC SPECIES
}

\author{
ESPÍNDOLA, E. L. G., ${ }^{1}$ MATSUMURA-TUNDISI, T., ${ }^{2}$ RIETZLER, A. C. ${ }^{3}$ and TUNDISI, J. G. ${ }^{2}$ \\ ${ }^{1}$ Departamento de Hidráulica e Saneamento, CRHEA, Escola de Engenharia de São Carlos, Universidade de \\ São Paulo, São Carlos, SP, Brazil \\ ${ }^{2}$ Instituto Internacional de Ecologia, São Carlos, SP, Brazil \\ ${ }^{3}$ Universidade Federal de Minas Gerais, Belo Horizonte, MG, Brazil \\ Correspondence to: Evaldo Luíz Gaeta Espíndola, Departamento de Hidráulica e Saneamento, CRHEA, \\ Escola de Engenharia de São Carlos, Universidade de São Paulo, São Carlos, SP, Brazil \\ Received November 9, 1998 - Accepted February 10, 2000 - Distributed May 31, 2000
}

(With 6 figures)

\begin{abstract}
With the purpose of analyzing the horizontal distribution of the zooplankton community of the Tucuruí Reservoir in the State of Pará, Brazil, collections were made at 16 stations during August 1988. The stations cover the regions called Caraipé (C), Araçagi (B) and Ararão (A), and represent the different compartments of this ecosystem in regard to the morphometry and the presence or absence of "flooded forest". Our findings showed the existence of three compartments with different limnological characteristics determined as a function of the system's morphometry, a factor that may have influenced the horizontal circulation of the reservoir's water and, consequently, led to physical, chemical and biological differences at each of the sampled stations. All the stations presented physical and chemical stratification and layers of total anoxia or reduced concentrations of oxygen dissolved at depths corresponding to the limit of the euphotic zone. With regard to the zooplankton, a differentiated distribution that was mainly quantitative was found among the compartments (density of organisms and proportion among the species) in the case of Cladocera and Copepoda, and basically qualitative in the case of Rotifera. On the other hand, the greatest densities of organisms for all the groups were recorded at the Caraipé stations. As for the spatial distribution, some species were more restricted or more abundant at given stations. Among the Cladocera, there was a greater abundance of Moina minuta, Bosmina hagmani and Bosminopsis deitersi at the Araçagi stations, while C. cornuta intermedia, $C$. cornuta rigaudi and C. cornuta cornuta were more plentiful in the Caraipé and Diaphanosoma birgei in the Ararão. Among the Rotifera, Trochosphaera aequatoriales and Lecane proiecta, Ascomorpha ecaudis, besides Polyartha cf vulgaris were restricted, respectively, to the Caraipé, Araçagi and Ararão stations, while the others, such as Keratella americana and Collotheca sp., were more broadly distributed. As regards Copepoda Calanoida, the dominating species was found to be Notodiaptomus maracaibenses, followed by Notodiaptomus henseni and Argyrodiaptomus azevedoi. The most abundant Cyclopoda species was Thermocyclops minutus, although Thermocyclops decipiens, Mesocyclops longisetus, Mesocyclops meridianus e Metacyclops sp. were also found.
\end{abstract}

Key words: zooplankton, horizontal distribution, spatial heterogeneity, reservoirs.

\section{RESUMO}

\section{Heterogeneidade espacial do Reservatório de Tucuruí (Estado do Pará, Amazônia, Brasil) e a distribuição das espécies zooplanctônicas}

Com o objetivo de analisar a distribuição horizontal da comunidade do zooplâncton no Reservatório de Tucuruí, Pará, foram efetuadas coletas em 16 estações durante o período de agosto de 1988. As estações 
compreenderam as regiões denominadas de Caraipé (C), Araçagi (B) e Ararão (A), as quais representam diferentes compartimentos desse ecossistema em relação à morfometria e à presença ou ausência da "mata afogada". Os resultados obtidos mostraram a existência de três compartimentos com distintas características limnológicas determinadas em função da morfometria do sistema, fator este que pode ter influenciado na circulação horizontal da água no reservatório e, conseqüentemente, levado a diferenças físicas, químicas e biológicas em cada estação amostrada. Todas as estações apresentaram estratificações física e química e camadas de total anoxia ou reduzida concentração de oxigênio dissolvido em profundidades correspondentes ao limite da zona eufótica. Em relação ao zooplâncton, verificou-se uma distribuição diferenciada entre os compartimentos, diferença esta principalmente quantitativa (densidade de organismos e proporção entre as espécies) no caso de Cladocera e Copepoda, enquanto essencialmente qualitativa no caso de Rotifera. Por outro lado, as maiores densidades de organismos para todos os grupos foram registradas nas estações do Caraipé. Quanto a distribuição espacial, algumas espécies mostraram-se restritas ou mais abundantes em determinadas estações. Entre os Cladocera, Moina minuta, Bosmina hagmani e Bosminopsis deitersi foram mais abundantes nas estações do Araçagi, C. cornuta intermedia, $C$. cornuta rigaudi e $C$. cornuta cornuta, no Caraipé e Diaphanosoma birgei, no Ararão. Entre Rotifera, Trochosphaera aequatoriales e Lecane proiecta, Ascomorpha ecaudis, além de Polyartha cf vulgaris, estiveram restritas às estações do Caraipé, Araçagi e Ararão, respectivamente, enquanto outras apresentaram distribuição mais ampla como Keratella americana e Collotheca sp. Quanto a Copepoda Calanoida, Notodiaptomus maracaibenses foi a espécie dominante, seguida de Notodiaptomus henseni e Argyrodiaptomus azevedoi. Thermocyclops minutus foi a espécie mais abundante dentre Cyclopoida, tendo sido também encontrados Thermocyclops decipiens, Mesocyclops longisetus, Mesocyclops meridianus e Metacyclops sp.

Palavras-chave: zooplâncton, distribuição horizontal de organismos, heterogeneidade espacial, reservatórios.

\section{INTRODUCTION}

Reservoirs are intermediate ecosystems between rivers and lakes. Besides being subject to the action of climatological forces such as precipitation, wind and solar radiation, are also closely related to the operational mechanisms of dams (outflow and retention time). These, together with the system's morphometry, produce differences in the horizontal and vertical circulation throughout a spatial gradient (compartmentalization), making them extremely dynamic environments with a high spatial and temporal variability of their physical and chemical characteristics (Tundisi, 1981; Tundisi, 1990; Armengol \& Saab, 1990) and producing differences in the qualitative and quantitative structural organization of their communities (Burgis, 1969; Watson \& Carpenter, 1974; Hart, 1978; Hayward \& Van Den Avyle, 1986; Cryer \& Townsend, 1988; Infante, 1995). Another factor to be considered is the input of nutrients via affluents, which become diluted toward the main axis, and the many secondary water sources along the hydrographic basin that flow into the reservoir and contribute not only to the eutrophication of the system but also to the formation of compartments with different environmental conditions. However, although they recognize the horizontal heterogeneity and the compartmentalization of the abiotic factors, most studies of biological communities are still carried out descriptively, evaluating the temporal distribution of communities in seasonal cycles, emphasizing their structural alterations (composition and density) or determining metabolic processes in a single season, all of which is information considered to be representative of the system under study (Stavn, 1971; Soto et al., 1984; Jones et al., 1995).

Organisms, present spatial and temporal variations related to the physical and chemical gradients imposed not only by the difference in the longitudinal axis produced by the transition from river to lake but also by the system's morphometric differences. Thus, in their natural environment, 
organisms are not distributed homogeneously and present considerable differences in their vertical and horizontal distribution, such as aggregated distribution (Hutchinson, 1967; Stavn, 1971).

Taking into account this spatial variability and the importance of the planktonic community in the food chain, we sought to characterize the distribution and spatial heterogeneity of the organisms in the Tucuruí (State of Pará) Reservoir, with emphasis on the zooplankton community and its relation to some environmental variables.

\section{AREA OF STUDY}

The Tucuruí hydroelectric power plant, formed by the damming of the Tocantins river, is the second largest hydroelectric plant established essentially in Brazilian territory, and is located in the State of Pará, at an altitude of $72 \mathrm{~m}$, between the latitudes of $3^{\circ} 45^{\prime}$ and $5^{\circ} 15^{\prime}$ 'South and longitudes $49^{\circ} 12^{\prime}$ and $50^{\circ} 00^{\prime}$ 'West. The basin's drainage area spreads over $758,000 \mathrm{~km}^{2}$ and the area of the reservoir is $2,430 \mathrm{~km}^{2}$, with a maximum width of $40 \mathrm{~km}$ and an average width of $14.3 \mathrm{~km}$. It is 170 $\mathrm{km}$ long and has a maximum depth of $75.0 \mathrm{~m}$ and average depth of $18.9 \mathrm{~m}$. The affluent inflow is $11,090 \mathrm{~m}^{3} / \mathrm{s}$, with an average residence time of 47.8 days. The magnitude of the outflow, according to information obtained by ELETRONORTE allows for the total renewal of the reservoir's waters in less than two months, since the Tocantins River's average outflow at Tucuruí is considered high, and has been recorded as $3,500 \mathrm{~m}^{3} / \mathrm{s}$ during the dry season and as $35,000 \mathrm{~m}^{3} / \mathrm{s}$ during the rainy season.

According to information from ELETRONORTE, a great phytomass was flooded as a result of the damming of the Tocantins waters, and this material is undergoing a gradual process of decomposition until its complete mineralization and humification owing to the fact that this process is taking place under a dense layer of inorganic sediments deriving from upstream erosion. Another important consequence was the formation of innumerable islands (around 600) around the borders of the reservoir, which, associated with or without prior deforestation, form different compartments in the system and contribute toward increased environmental heterogeneity.

\section{MATERIAL AND METHODS}

Due to the reservoir's size, the collections were made in areas closer to the dam, considering two principal aspects: the presence or absence of previously deforested areas and the system's morphometry (the presence or absence of islands). The collections were carried out during the dry season on August 24, 25 and 26, 1988 at 16 stations called the Caraipé (from $\mathrm{C}_{1}$ to $\mathrm{C}_{7}$ ), Araçagi (from $\mathrm{B}_{1}$ to $\mathrm{B}_{5}$ ) and Ararão (from $\mathrm{A}_{1}$ to $\mathrm{A}_{4}$ ) stations, as shown in Fig. 1.

The zooplankton samples were collected by means of vertical dragging in the water column, with the material concentrated in a $68 \mathrm{~mm}$ sized mesh plankton net. The material collected was fixed with $4 \%$ formalin and analyzed in a laboratory, considering the composition and density of the organisms. Identification of the organisms, including the Cladocera, Copepoda (Cyclopoida and Calanoida) and Rotifera species, was carried out based on the following references: Pennak (1953), Edmondson (1959), Smirnov (1974), Smirnov \& Timms (1983), Rocha \& Matsumura-Tundisi (1976), Koste (1978a, 1978b), Reid (1985) and Matsumura-Tundisi $(1984,1986)$, Montú \& Goeden (1986). The density of the organisms was determined by counting the sub-samples (enumerating at least 100 organisms of the most abundant species) or by total sample, as a function of organism density in each sample. Quantification of the rotifers was carried out by Sedgwick-Rafter slide while the density of the copepods and cladocerans was determined using the methods of Edmondson \& Winberg (1971). The Copepoda, particularly, were analyzed considering the nauplius, copepodit and adult phases (males, females and spawning females).

Other organisms present in the samples were also included in the analysis, such as insect larvae, alevins, turbellarians and ostracods. The findings were expressed in numbers of individuals per $\mathrm{m}^{-3}$. The volume of water filtered by the net was calculated according to Edmondson \& Winberg (1971).

The indexes of species diversity was calculated based on the composition and density data, using the Shannon \& Weaver (1949) index, 


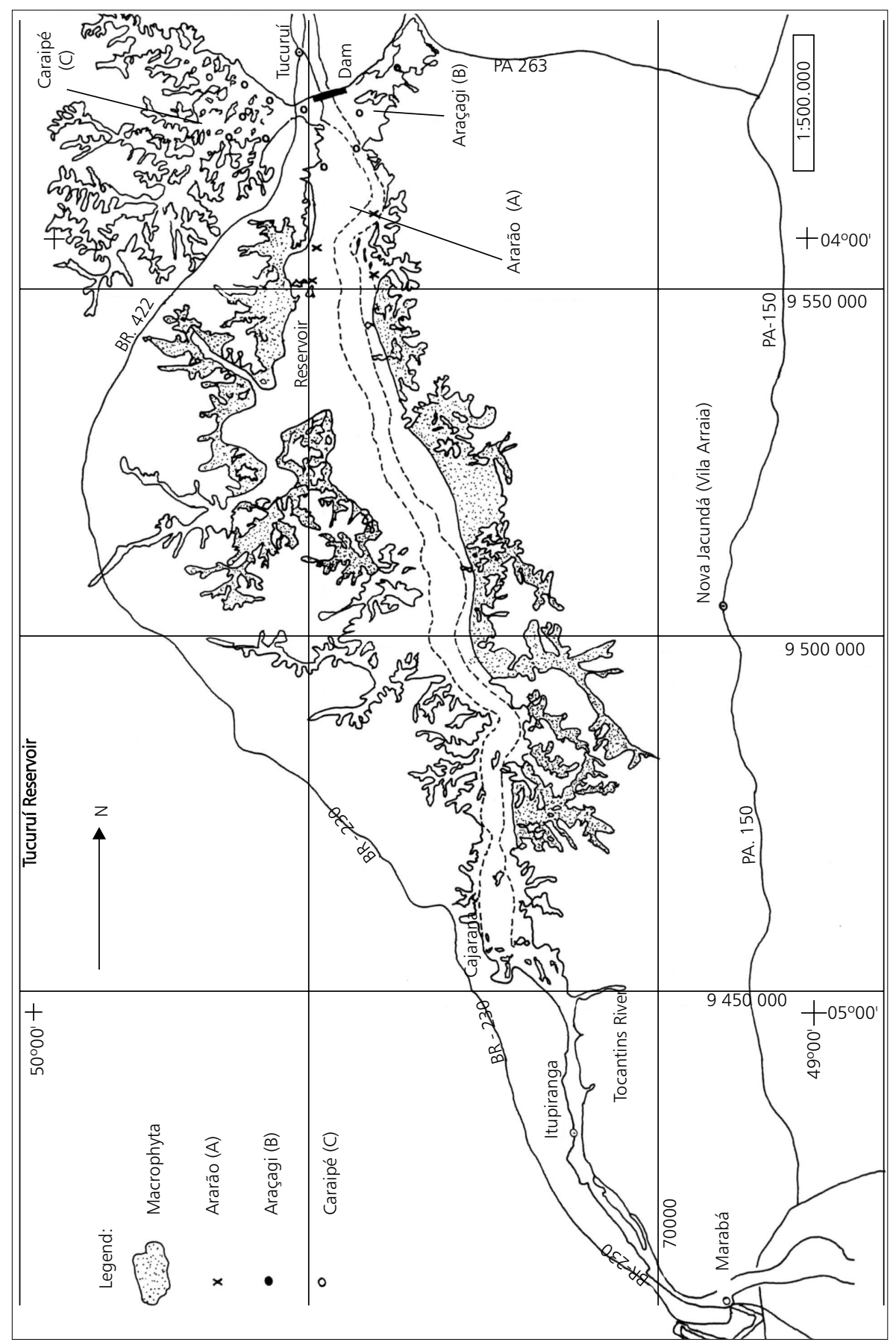

Fig. 1 - Morfometry of the Tucuruí Reservoir and the locations of the Caraipé, Araçagi and Ararão stations. 
while the index of similarity was calculated using Sorensen's Index of Similarity (1948).

To evaluate the plankton biomass (in terms of dry weight), samples were collected be vertical dragging using a $68 \mathrm{~mm}$ mesh plankton net. The material thus collected was filtered using a MILLIPORE AP20 filter (with $47 \mathrm{~mm}$ diameter e 0,45 $\mathrm{mm}$ hole pore openings), previously calcined in a muffler at $460^{\circ} \mathrm{C}$ for 2 hours and weighed to reach Weight 01 . After filtering, the filters were left in an oven at $60^{\circ} \mathrm{C}$ for 24 hours, after which they were weighed to find the total weight of the material, determined as Weight 02 , using the difference in weight to estimate the dry weight of the organic material.

Water samples were collected at three depths (surface, limit of the euphotic zone and aphotic zone) to determine the $\mathrm{pH}$ (using a MICRONAL B382 potentiometer), conductivity (using a DIGIMED CD-20 conductivimeter), dissolved oxygen (using the Winkler method described in Golterman et al., 1978) and chlorophyll $a+$ phaeopigments (Golterman et al., 1978). Measurements were also made of the local depth, water temperature profiles (using a Toho Dentan thermistor), water transparency (by Secchi disc) and the extent of the euphotic zone (defined as the point of disappearance of the Secchi disc multiplied by three, according to Esteves, 1988).

\section{RESULTS}

\section{Limnological characterization of the Tucurui Reservoir}

The findings of some of the limnological variables summarized in Table 1 indicate that the $\mathrm{pH}$ and conductivity values of the sampled stations did not differ significantly. The $\mathrm{pH}$ values found for Caraipé lay between 6.59 and 7.06, those of Araçagi ranged from 6.94 to 7.22 and those of Ararão were between 7.06 to 7.14 , while conductivity values were from 47.6 to $52.7 \mu \mathrm{S} . \mathrm{cm}^{-1}$ at Caraipé, 47.6 to $50.2 \mu \mathrm{S} . \mathrm{cm}^{-1}$ at Araçagi and 52.1 to 53.0 $\mu \mathrm{S} . \mathrm{cm}^{-1}$ at Ararão. However, a vertical chemical gradient was found at all the stations, with lower $\mathrm{pH}$ values and higher conductivity values at depths corresponding to the aphotic zone. The dissolved oxygen concentrations varied from 3.90 to 4.99 $\mu \mathrm{g} . \mathrm{L}^{-1}$ at Caraipé, from 4.82 to $5.69 \mu \mathrm{g} . \mathrm{L}^{-1}$ at Araçagi and from 4.90 to $5.0 \mu \mathrm{g} . \mathrm{L}^{-1}$ at Ararão, and layers of total anoxia were found at most of the stations. With regard to water transparency, lower values were found at the Caraipé stations due to the high contribution of organic material, leading to a reduction of the euphotic zone at these sites. The temperature profiles showed a difference of $4^{\circ} \mathrm{C}$ between the surface and the bottom at the Caraipé stations and of $2.4^{\circ} \mathrm{C}$ and $2.6^{\circ} \mathrm{C}$, respectively, at the Araçagi and Ararão stations.

The variation in chlorophyll a concentrations, considering the mean values between the depths sampled (Fig. 2), showed a greater phytoplanktonic biomass at the Caraipé stations (between 3.73 and $9.41 \mu \mathrm{g} . \mathrm{L}^{-1}$ ), with values ranging from 3.22 to 4.28 $\mu \mathrm{g} . \mathrm{l}^{-1}$ at the Araçagi and from 3.11 to $4.02 \mu \mathrm{g} . \mathrm{L}^{-1}$ at the Ararão stations. The planktonic biomass, estimated in dry weight (Fig. 3) was also higher at the Caraipé stations, with values varying from 5.85 to $35.93 \mathrm{mg} . \mathrm{m}^{-3}$. The variation at the Araçagi stations ranged from 5.40 to $17.02 \mathrm{mg} . \mathrm{m}^{-3}$, while at the Ararão stations it was between 9.34 and $25.97 \mathrm{mg} . \mathrm{m}^{-3}$.

\section{Composition, abundance and dominance of the zooplankton community}

Table 2 shows the composition and density (ind $/ \mathrm{m}^{3}$ ) of zooplankton species found at the 16 stations analyzed. Thirty-four species of Rotifera, 15 species of Cladocera and 8 species of Copepoda were found, besides Turbellaria, Ostracoda and Chaoborus.

The dominance of the Copepoda group over the Rotifera was observed at the all stations. Table 3 summarizes the relative abundance of the most common zooplankton groups in the samples taken from the three sites, Araçagi, Ararão and Caraipé. There was an evident dominance of copepods at all the sites, particularly those of the Ararão stations, where this group's dominance was $70.7 \%$. However, the highest density was found at the Caraipé stations.

The Caraipe stations were dominated by the Cladocera species Ceriodaphnia cornuta (87\%) in the following three varieties: cornuta, intermedia and righaudi and by Diaphanosoma birgei $(15.9 \%)$. The stations of Ararão were also dominated by Ceriodaphnia cornuta (67\%), but only two of its forms: cornuta and intermedia. 
TABLE 1

Depth, secchi, temperature, pH, conductivity and dissolved oxygen found at the three locations (Caraipé, Araçagi and Ararão) in the Tucuruí Reservoir.

\begin{tabular}{|c|c|c|c|c|c|}
\hline Station - Depth. (m) & Secchi (m) e Depth. (m) & Temp ("C) & $\mathbf{p H}$ & Cond. $\left(\mu\right.$ S.cm $\left.{ }^{-1}\right)$ & DO $\left(\mathrm{mg} . \mathrm{L}^{-1}\right)$ \\
\hline $\begin{array}{c}\mathrm{C} 1-0.0 \\
5.0 \\
10.0\end{array}$ & $3.30-(24.0)$ & $26.8-30.8$ & $\begin{array}{l}6.52 \\
6.48 \\
6.77 \\
\end{array}$ & $\begin{array}{l}48.5 \\
52.6 \\
48.9\end{array}$ & $\begin{array}{l}7.22 \\
4.14 \\
0.35\end{array}$ \\
\hline $\begin{array}{c}\mathrm{C} 2-0.0 \\
4.0 \\
8.0\end{array}$ & $3.00-(18.0)$ & $28.4-31.4$ & $\begin{array}{l}7.12 \\
6.97 \\
6.66\end{array}$ & $\begin{array}{l}48.6 \\
48.6 \\
49.5\end{array}$ & $\begin{array}{l}7.05 \\
5.46 \\
0.44\end{array}$ \\
\hline $\begin{array}{c}\mathrm{C} 3-0.0 \\
4.0 \\
8.0\end{array}$ & $3.10-(27.0)$ & $27.5-30.8$ & $\begin{array}{l}7.05 \\
7.06 \\
6.62 \\
\end{array}$ & $\begin{array}{l}47.7 \\
48.0 \\
48.7 \\
\end{array}$ & $\begin{array}{l}6.61 \\
6.61 \\
0.79 \\
\end{array}$ \\
\hline $\begin{array}{c}\mathrm{C} 4-0.0 \\
3.5 \\
7.0\end{array}$ & $2.50-(23.0)$ & $27.5-31.2$ & $\begin{array}{l}7.16 \\
7.20 \\
6.70 \\
\end{array}$ & $\begin{array}{l}47.9 \\
47.8 \\
47.2 \\
\end{array}$ & $\begin{array}{l}7.31 \\
7.05 \\
0.53\end{array}$ \\
\hline $\begin{array}{c}\mathrm{C} 5-0.0 \\
4.0 \\
8.0 \\
\end{array}$ & $3.00-(28.0)$ & $27.0-29.6$ & $\begin{array}{l}7.14 \\
7.21 \\
6.84 \\
\end{array}$ & $\begin{array}{l}48.0 \\
48.5 \\
48.8 \\
\end{array}$ & $\begin{array}{l}5.73 \\
7.22 \\
2.03\end{array}$ \\
\hline $\begin{array}{c}\mathrm{C} 6-0.0 \\
4.0 \\
8.0 \\
15.0 \\
\end{array}$ & $3.10-(21.0)$ & $28.3-30.8$ & $\begin{array}{l}7.21 \\
7.20 \\
6.88 \\
6.52 \\
\end{array}$ & $\begin{array}{l}49.0 \\
49.2 \\
50.6 \\
62.0 \\
\end{array}$ & $\begin{array}{l}7.84 \\
7.14 \\
2.29 \\
0.00 \\
\end{array}$ \\
\hline $\begin{array}{c}C 7-0.0 \\
4.0 \\
8.0 \\
15.0 \\
\end{array}$ & $3.00-(40.0)$ & $27.9-30.2$ & $\begin{array}{l}7.08 \\
7.10 \\
6.90 \\
6.67\end{array}$ & $\begin{array}{l}48.7 \\
48.3 \\
49.6 \\
54.5\end{array}$ & $\begin{array}{l}7.66 \\
7.22 \\
3.44 \\
0.35 \\
\end{array}$ \\
\hline $\begin{array}{c}\mathrm{A} 1-0.0 \\
6.0 \\
12.0\end{array}$ & $4.70-(45.0)$ & $28.4-29.5$ & $\begin{array}{l}7.26 \\
7.14 \\
6.78\end{array}$ & $\begin{array}{l}51.6 \\
51.2 \\
53.5\end{array}$ & $\begin{array}{l}7.22 \\
6.99 \\
0.79\end{array}$ \\
\hline $\begin{array}{c}\mathrm{A} 2-0.0 \\
7.0 \\
15.0\end{array}$ & $5.75-(21.0)$ & $28.5-29.8$ & $\begin{array}{l}7.31 \\
7.26 \\
6.87 \\
\end{array}$ & $\begin{array}{l}52.9 \\
53.0 \\
53.0\end{array}$ & $\begin{array}{l}7.22 \\
7.05 \\
1.14 \\
\end{array}$ \\
\hline $\begin{array}{c}\mathrm{A} 3-0.0 \\
7.0 \\
15.0 \\
\end{array}$ & $5.70-(37.0)$ & $28.3-30.2$ & $\begin{array}{l}7.26 \\
7.26 \\
6.95 \\
\end{array}$ & $\begin{array}{l}52.5 \\
53.3 \\
53.2 \\
\end{array}$ & $\begin{array}{l}6.87 \\
6.61 \\
1.23 \\
\end{array}$ \\
\hline $\begin{array}{c}\mathrm{A} 4-0.0 \\
6.0 \\
12.0\end{array}$ & $4.30-(38.0)$ & $28.2-30.8$ & $\begin{array}{l}7.20 \\
7.20 \\
6.97 \\
\end{array}$ & $\begin{array}{l}52.6 \\
52.5 \\
52.5\end{array}$ & $\begin{array}{l}6.78 \\
6.16 \\
2.20 \\
\end{array}$ \\
\hline $\begin{array}{c}\mathrm{B} 1-0.0 \\
6.0 \\
12.0\end{array}$ & $4.30-(15.0)$ & $28.6-29.5$ & $\begin{array}{l}7.43 \\
7.40 \\
6.83 \\
\end{array}$ & $\begin{array}{l}48.8 \\
49.0 \\
49.7 \\
\end{array}$ & $\begin{array}{l}7.84 \\
7.49 \\
1.76 \\
\end{array}$ \\
\hline $\begin{array}{c}\mathrm{B} 2-0.0 \\
6.0 \\
12.0 \\
20.0\end{array}$ & $4.10-60.0)$ & $28.2-29.5$ & $\begin{array}{l}7.36 \\
7.27 \\
6.96 \\
6.86 \\
\end{array}$ & $\begin{array}{l}49.4 \\
49.5 \\
49.6 \\
52.5 \\
\end{array}$ & $\begin{array}{l}7.57 \\
7.22 \\
3.26 \\
2.20\end{array}$ \\
\hline $\begin{array}{c}\text { B3 }-0.0 \\
7.0 \\
15.0 \\
20.0 \\
\end{array}$ & $5.40-(76.0)$ & $28.3-29.2$ & $\begin{array}{l}7.25 \\
7.25 \\
7.10 \\
6.85\end{array}$ & $\begin{array}{l}49.8 \\
49.7 \\
50.3 \\
50.5 \\
\end{array}$ & $\begin{array}{l}7.22 \\
6.87 \\
4.49 \\
2.03\end{array}$ \\
\hline $\begin{array}{c}\mathrm{B} 4-0.0 \\
6.0 \\
12.0\end{array}$ & $4.20-(17.0)$ & $28.5-30.8$ & $\begin{array}{l}7.09 \\
6.95 \\
6.80 \\
\end{array}$ & $\begin{array}{l}47.0 \\
48.2 \\
47.6 \\
\end{array}$ & $\begin{array}{l}6.08 \\
4.40 \\
2.29\end{array}$ \\
\hline $\begin{array}{c}\mathrm{B} 5-0.0 \\
5.0 \\
11.0 \\
15.0\end{array}$ & $4.00-(29.0)$ & $28.2-30.6$ & $\begin{array}{l}7.24 \\
7.13 \\
6.97 \\
6.84 \\
\end{array}$ & $\begin{array}{l}48.0 \\
49.0 \\
48.6 \\
48.0\end{array}$ & $\begin{array}{l}7.22 \\
5.72 \\
3.70 \\
2.64\end{array}$ \\
\hline
\end{tabular}


TABLE 2

Composition, abundance (Ind.m ${ }^{-3}$ ) and distribution of the zooplankton in the Tucuruí Reservoir.

\begin{tabular}{|c|c|c|c|c|c|c|c|c|c|c|c|c|c|c|c|c|}
\hline Species & C1 & $\mathrm{C2}$ & C3 & $\mathrm{C4}$ & C5 & C6 & C7 & $\mathbf{A 1}$ & $\mathbf{A 2}$ & $\mathbf{A 3}$ & A4 & B1 & B2 & B3 & B4 & B5 \\
\hline \multicolumn{17}{|l|}{ CLADOCERA } \\
\hline Alona retangula pulchra & - & - & - & - & - & - & - & 2 & 1 & 1 & 1 & - & - & - & - & - \\
\hline Bosminopsis deiters & 169 & 110 & 142 & 113 & 235 & 122 & 70 & 421 & 8 & 14 & 2 & 765 & 205 & 143 & 232 & 441 \\
\hline Bosmina hagman & 51 & 91 & 60 & 80 & 190 & 85 & 20 & 32 & 16 & 7 & 6 & 296 & 29 & 23 & 192 & 240 \\
\hline C. cornuta rigaud & 90 & 85 & 124 & 97 & 120 & 106 & 201 & 5 & 1 & 5 & 5 & 21 & 4 & 8 & 70 & 192 \\
\hline C. cornuta intermedia & 54 & 173 & 461 & 250 & 369 & 130 & 16 & 29 & 2 & 4 & 14 & 29 & 4 & 4 & 50 & 74 \\
\hline C. cornuta cornuta & 750 & 100 & 58 & 118 & 449 & 263 & 44 & 515 & 100 & 75 & 204 & 115 & 28 & 23 & 129 & 182 \\
\hline Ceriodaphnia reticulata & - & - & 6 & - & - & 8 & $\begin{array}{lll}- & & \\
\end{array}$ & - & - & - & - & - & $\begin{array}{lll}- & & \\
-\end{array}$ & $\begin{array}{l}- \\
-\end{array}$ & - & $\begin{array}{cc}- & \\
\end{array}$ \\
\hline Diaphanosoma birgei & 64 & 396 & 157 & 240 & 490 & 481 & 83 & 399 & 120 & 94 & 71 & 346 & 53 & 98 & 53 & 79 \\
\hline Diaphanosoma spinulosum & - & 65 & 16 & 17 & 21 & 17 & 1 & - & 4 & 13 & 15 & 23 & 3 & 12 & 3 & - \\
\hline Daphnia gessneri & 90 & 28 & 1 & - & 7 & 8 & - & 2 & - & - & 98 & - & - & - & - & - \\
\hline Grimaldina brazza & - & $\begin{array}{llll}- & & & 0\end{array}$ & - & - & - & - & - & - & - & - & $\begin{array}{llll}- & & & 0\end{array}$ & 3 & - & - & - & - \\
\hline Ilyocryptus spinifer & $\begin{array}{l}- \\
\end{array}$ & - & $\begin{array}{ll}- & \\
\end{array}$ & $\begin{array}{l}- \\
\end{array}$ & - & $\begin{array}{l}- \\
\end{array}$ & - & - & 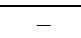 & - & 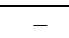 & - & 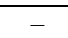 & - & 1 & - \\
\hline Latonopsis fasciculata & - & - & - & - & - & - & - & - & 3 & 5 & 22 & - & - & - & - & - \\
\hline Moina minuta & 25 & 11 & 63 & 50 & 59 & 7 & 20 & 8 & 7 & 14 & 1 & 403 & 13 & 38 & 140 & 155 \\
\hline Simoceplallus serrulatus & 14 & 3 & - & - & - & - & - & - & 1 & 8 & - & - & - & - & - & \\
\hline \multicolumn{17}{|l|}{ COPEPODA } \\
\hline Notodiaptomus henseni & 492 & 448 & 599 & 345 & 52 & 116 & 7 & 368 & 120 & 115 & 303 & 140 & 19 & 18 & 15 & 14 \\
\hline N. maracaibensis & 474 & 283 & 453 & 204 & 61 & 94 & 27 & 423 & 207 & 130 & 433 & 176 & 47 & 41 & 53 & 28 \\
\hline Argyrodiaptomus azevedoi & 56 & 41 & 53 & 4 & - & 6 & 34 & 1 & 2 & 1 & 4 & - & 7 & 2 & 2 & 2 \\
\hline Thermocyclops minutus & 514 & 771 & 939 & 360 & 144 & 171 & 477 & 145 & 810 & 563 & 104 & 212 & 401 & 606 & 205 & 115 \\
\hline T. decipiens & 4 & - & - & 5 & 5 & - & - & - & 6 & 10 & 9 & 2 & 6 & 3 & - & 1 \\
\hline Mesocyclops meridianus & - & - & - & 1 & - & - & - & - & - & - & - & 2 & - & - & - & - \\
\hline$M$. longisetus & 2 & - & - & - & 2 & 2 & - & - & 2 & - & 1 & 4 & 2 & - & - & - \\
\hline Metacyclops sp. & - & - & - & - & - & - & - & - & - & - & - & 10 & - & - & - & - \\
\hline Nauplii of Calanoida & 467 & 956 & 644 & 917 & 536 & 102 & 314 & 103 & 978 & 373 & 632 & 597 & 348 & 299 & 607 & 509 \\
\hline Nauplii of Cyclopoida & 303 & 397 & 365 & 297 & 428 & 619 & 189 & 367 & 288 & 123 & 924 & 355 & 563 & 214 & 294 & 275 \\
\hline Copepodites of Calanoida & 347 & 478 & 239 & 422 & 237 & 180 & 88 & 775 & 669 & 477 & 622 & 220 & 202 & 163 & 308 & 253 \\
\hline Copepodites of Cyclopoida & 574 & 127 & 126 & 773 & 129 & 123 & 283 & 541 & 502 & 477 & 538 & 283 & 216 & 362 & 661 & 473 \\
\hline \multicolumn{17}{|l|}{ ROTÍFERA } \\
\hline Ascomorpha ecaudis & - & - & - & - & - & - & - & - & - & 4 & - & 268 & 19 & 44 & - & 35 \\
\hline Ascomorpha ovalis & 16 & 28 & 91 & - & - & - & 14 & - & 25 & 53 & 41 & - & 33 & - & 57 & - \\
\hline Ascomorpha saltans & 25 & 28 & 10 & 10 & - & - & 4 & 8 & 50 & 12 & 7 & 26 & 5 & 29 & 57 & 11 \\
\hline Asplanchna $\mathrm{sp}$. & - & 42 & 61 & - & - & - & - & - & - & - & - & - & - & - & 38 & - \\
\hline Bdelloidea $\mathrm{sp}$. & - & - & - & - & - & - & - & 46 & - & - & - & - & - & - & - & - \\
\hline Brachionus dolabratus & 8 & 99 & 71 & 38 & 128 & - & 32 & - & - & - & - & - & - & - & 160 & 70 \\
\hline B. falcatus & 16 & $\begin{array}{llll}- & & & l\end{array}$ & 20 & 10 & 14 & 85 & $\begin{array}{llll}- & & & \end{array}$ & - & - & - & - & - & - & - & 9 & 35 \\
\hline
\end{tabular}




\begin{tabular}{|c|c|c|c|c|c|c|c|c|c|c|c|c|c|c|c|c|}
\hline $\begin{array}{r}\text { Species } \\
\end{array}$ & C1 & C2 & C3 & $\mathrm{C4}$ & C5 & C6 & C7 & A1 & A2 & A3 & A4 & B1 & B2 & B3 & B4 & B5 \\
\hline B. patulus & 8 & 14 & 51 & - & 235 & 66 & 36 & - & - & - & - & - & - & - & - & - \\
\hline B. mirus angustu & - & 14 & - & 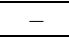 & - & 19 & 4 & - & - & - & - & 38 & - & 37 & 207 & 224 \\
\hline B. zahniseri & $\begin{array}{l}- \\
-\end{array}$ & 28 & 30 & 10 & 36 & 28 & 7 & $\begin{array}{l}- \\
-\end{array}$ & $\begin{array}{l}- \\
-\end{array}$ & - & $\begin{array}{ll}- \\
\end{array}$ & 281 & 104 & 124 & 415 & 271 \\
\hline Collotheca sp. & 58 & 198 & 304 & 76 & 156 & 255 & 128 & 39 & 6 & 114 & 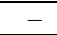 & 38 & 5 & 11 & 170 & 141 \\
\hline Conochilus coenobasi & $\begin{array}{llll}- & & & 0\end{array}$ & 28 & 61 & 10 & 29 & 28 & 7 & 69 & 38 & 29 & 61 & 13 & $\begin{array}{l}- \\
\end{array}$ & $\begin{array}{llll}- & & & \\
\end{array}$ & 19 & 23 \\
\hline C. unicorni & - & - & 20 & - & - & 28 & - & 69 & 38 & 8 & 34 & - & - & - & 85 & 35 \\
\hline Filinia longiseta & - & 42 & 30 & - & 29 & - & 11 & - & - & - & - & - & - & 4 & 19 & 23 \\
\hline F. terminalis & 8 & 14 & 91 & 10 & 78 & 57 & 29 & 8 & - & - & - & - & - & - & - & - \\
\hline Horaella sp. & - & - & - & - & - & - & - & 31 & - & - & - & 38 & 24 & 37 & 57 & - \\
\hline Hexarthra int. brasiliensis & 16 & 28 & 51 & 19 & 14 & 38 & - & 46 & 25 & 41 & 62 & 383 & 85 & 44 & 217 & 247 \\
\hline Keratella americana & 8 & 14 & 51 & 28 & 114 & 113 & 50 & 8 & 44 & 61 & 62 & 217 & 19 & 26 & 566 & 354 \\
\hline K. lenzi & 16 & 28 & 30 & - & 43 & 47 & 50 & 23 & - & 12 & 21 & - & 28 & 26 & 57 & 23 \\
\hline K. paludosa & - & 14 & - & - & 7 & 9 & - & 8 & - & 20 & 14 & - & - & - & - & - \\
\hline Lecane arcuata & - & - & - & - & - & - & - & - & - & 4 & 7 & - & - & 4 & - & - \\
\hline L. chankensis & - & - & - & $\begin{array}{c}- \\
\end{array}$ & - & - & $\begin{array}{l}- \\
\end{array}$ & $\begin{array}{l}- \\
\end{array}$ & $\begin{array}{l}- \\
\end{array}$ & - & & - & - & $\begin{array}{l}- \\
\end{array}$ & $\begin{array}{l}- \\
\end{array}$ & - \\
\hline L. hamata & - & - & 30 & 28 & 57 & 19 & 28 & 8 & - & 4 & - & 13 & - & - & - & - \\
\hline L. proiecta & 66 & 297 & 637 & 86 & 856 & 405 & 264 & 8 & - & - & - & - & - & - & - & - \\
\hline Machrochaetus serricus & - & - & - & - & - & - & - & - & 6 & - & - & - & - & - & - & - \\
\hline Polyathra vulgaris & - & - & - & - & - & - & - & 100 & 19 & 253 & 82 & 89 & 156 & 95 & 85 & 130 \\
\hline Ptygura sp. & - & 42 & - & 10 & - & - & 7 & 31 & 13 & 65 & 96 & - & - & - & 9 & 23 \\
\hline Sinantherina socialis & 8 & 28 & - & - & 14 & - & 7 & 54 & - & 16 & 21 & - & - & - & 28 & - \\
\hline Synchaeta stylata & 25 & 99 & 51 & - & 14 & - & 11 & - & 13 & 4 & 75 & - & 14 & - & 38 & 23 \\
\hline Testudinella sp. & - & 28 & 40 & 10 & 107 & 47 & 39 & - & - & 82 & 41 & 153 & 14 & 11 & - & 23 \\
\hline Trichocerca sp. & 8 & - & 20 & - & 7 & - & 7 & - & 13 & - & - & - & 9 & 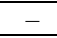 & 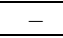 & 12 \\
\hline Trichocerca chattoni & 25 & 28 & 20 & 19 & 29 & 75 & 28 & 15 & 13 & 25 & 27 & 178 & 38 & 26 & 47 & 106 \\
\hline $\begin{array}{l}\text { Trochosphaera } \\
\text { aequatorialis }\end{array}$ & 712 & 240 & 384 & 601 & 14 & 274 & 7 & - & 6 & - & - & - & - & - & - & - \\
\hline Vonoyella globosa & 8 & 14 & - & 10 & 21 & - & - & - & - & 4 & - & - & - & - & - & - \\
\hline \multicolumn{17}{|l|}{ OTHER ORGANISMS } \\
\hline Chaoborus & 3 & 3 & 2 & 7 & 10 & 3 & 2 & - & - & 2 & 3 & - & - & 1 & 1 & 1 \\
\hline Turbellari & 6 & 11 & 1 & 3 & 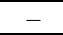 & 37 & $\begin{array}{l}- \\
\end{array}$ & - & - & 1 & 5 & - & - & 1 & 1 & 2 \\
\hline Ostracoda & 6 & 25 & 83 & 167 & 17 & 14 & 4 & - & - & - & 3 & 5 & 1 & - & 1 & - \\
\hline
\end{tabular}




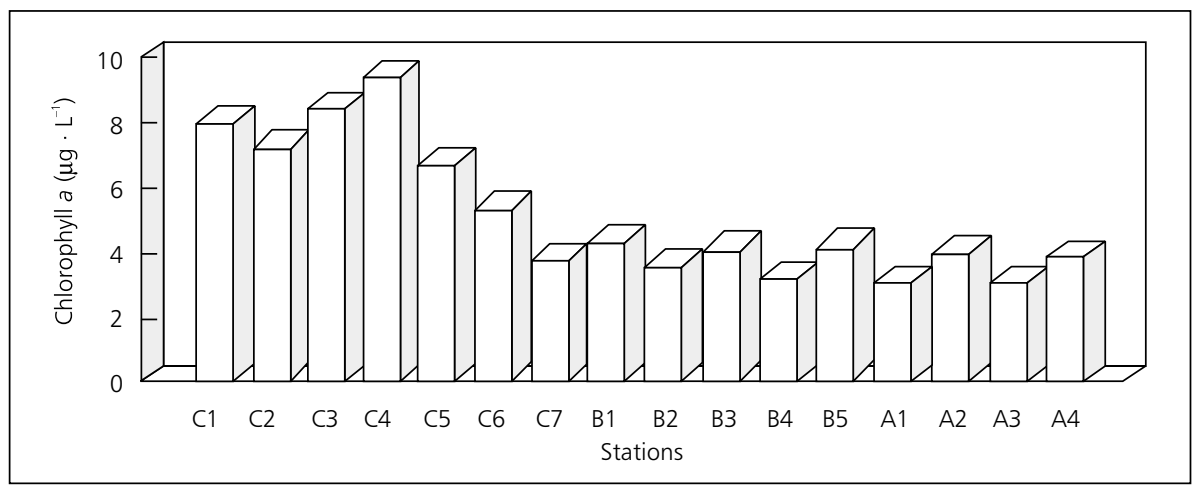

Fig. 2 - Chlorophyll $a$ concentration at the Caraipé (C), Araçagi (B) and Ararão (A) stations in the Tucuruí Reservoir.

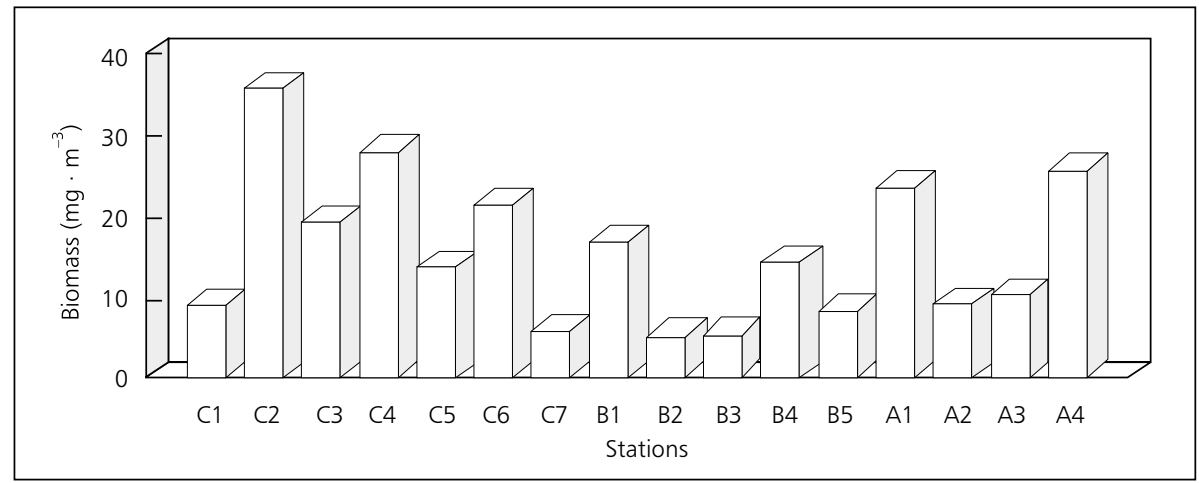

Fig. 3 - Biomass of the zooplankton at the Caraipé (C), Araçagi (B) and Ararão (A) stations in the Tucuruí Reservoir.

TABLE 3

Average values for copepods, cladocerans and rotifers (excluding nauplii) for the stations of each site.

\begin{tabular}{|c|c|c|c|c|c|c|}
\hline & \multicolumn{2}{|c|}{ Araçagi } & \multicolumn{2}{c|}{ Ararão } & \multicolumn{2}{c|}{ Caraipé } \\
\hline Groups & $\mathbf{I n d} / \mathbf{m}^{\mathbf{3}}$ & $\mathbf{\%}$ & $\mathbf{I n d} / \mathbf{m}^{\mathbf{3}}$ & $\mathbf{\%}$ & $\mathbf{I n d} / \mathbf{m}^{\mathbf{3}}$ & $\mathbf{\%}$ \\
\hline Copepoda (adults + copepodits) & 2,030 & 46.1 & 2,832 & 70.7 & 3,121 & 49.9 \\
\hline Cladocer & 984 & 22.4 & 589 & 14.7 & 1,722 & 27.5 \\
\hline Rotifer & 1,388 & 31.5 & 585 & 14.6 & 1,417 & 22.6 \\
\hline Total & 4,402 & & 4,006 & & 6,260 & \\
\hline
\end{tabular}

The Araçagi stations were dominated by three species: Bosminopsis deitersi $(36.3 \%)$, Bosmina hagmani (15.8\%) and Diaphanosoma birgei (12.8\%).

Fig. 4 shows the distribution of the most abundant species of Cladocera along the reservoir.
It can be seen that, among the varieties of Ceriodaphnia cornuta, the cornuta form dominated at some of the Caraipé stations and at all the Ararão stations, whereas the other two forms, righaudi and intermedia, were present in significant numbers only at the Caraipé stations. 

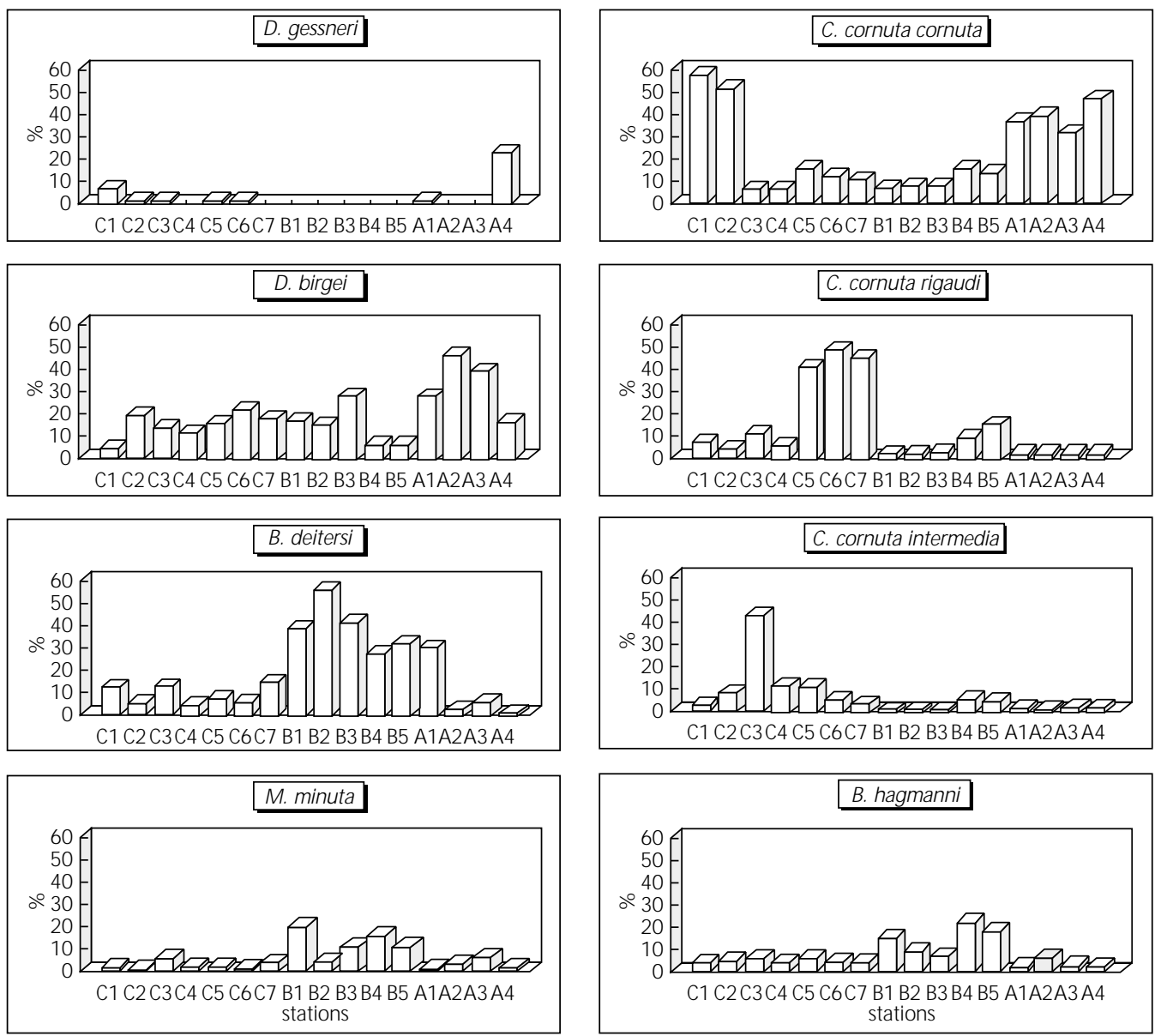

Fig. 4 - Horizontal distribution of the most abundant Cladocera species in the Tucuruí Reservoir $(\mathrm{C}=\mathrm{Caraipé}, \mathrm{B}=$ Araçagi and $\mathrm{A}=$ Ararão).

Bosminopsis deitersi was characteristic of the Araçagi stations. Other species such as Diaphanosoma birgei, Bosmina hagmani and Moina minuta were evenly distributed throughout the reservoir. Daphnia gessneri was present at some of the Caraipé stations and at two of the Ararão stations, but in very reduced numbers.

Some of the Rotifera species, such as Lecane proiecta and Trocosphaera aequatoriales, predominated at the Caraipé stations, while Polyarthra vulgaris and Keratella americana predominated at the Araçagi and Ararão stations. As can be observed in Fig. 5, Collotheca sp. was distributed homogeneously at every station of the three sites, although Ascomorpha aecaudis was restricted to the Araçagi stations. At the Ararão stations, besides the dominance of Keratella americana, two other species were also found to dominate: Hexarthra intermedia brasiliensis and Brachionus zahniseriu, the latter typical of the Amazonian region. The density of Rotifera was highest at the Araçagi stations (517 to 2,339 ind. $\left.\mathrm{m}^{-3}\right)$. The other sites showed densities ranging from 769 to 2,004 ind. $\mathrm{m}^{-3}$ (Caraipé) and from 308 to 813 ind. $\mathrm{m}^{-3}$ (Ararão). Cyclopoida dominated over Calanoida at all the stations of the three sites. This domination was more evident at Araçagi, where Cyclopoida represented $91.8 \%$. Table 4 shows the relative abundance of Cyclopoida and Calanoida at the three sites. 

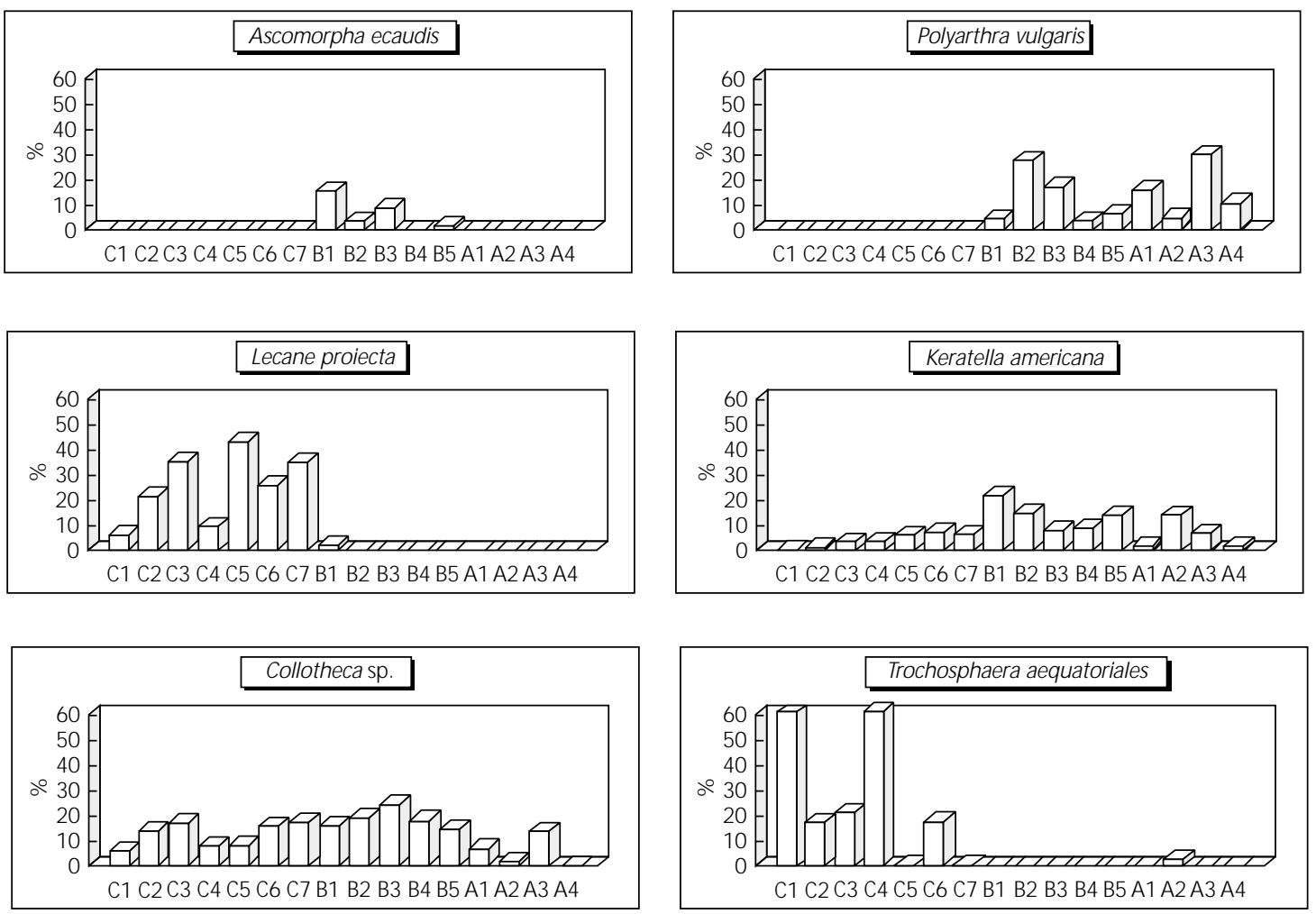

Fig. 5 - Horizontal distribution of the most abundant Rotifera species in the Tucuruí Reservoir $(\mathrm{C}=$ Caraipé, $\mathrm{B}=\mathrm{Araçagi}$ and $\mathrm{A}=$ Ararão).

TABLE 4

Relative abundance of Cyclopoida and Calanoida at the Caraipé, Ararão and Araçagi stations (only adult stages were considered).

\begin{tabular}{|c|c|c|c|}
\hline & Caraipé & Ararão & Araçagi \\
\hline Calanoida & $38.2 \%$ & $31.1 \%$ & $1.2 \%$ \\
\hline Cyclopoida & $61.8 \%$ & $68.9 \%$ & $91.8 \%$ \\
\hline
\end{tabular}

Thermocyclops minutus was the dominant Cyclopoida species. Other species such as Thermocyclps decipiens, Mesocyclops longisetus and Metacyclops sp occurred, albeit in much fewer numbers. With regard to Calanoida, three species were present: Notodiaptomus maracaibensis, Notodiaptomus henseni and Argyrodiaptomus azevedoi. At the Caraipé stations, the dominant species was Notodiaptomus henseni, while at the Ararão and Araçagi stations, Notodiaptomus maracaibensis predominated. Fig. 6 shows the distribution of the dominant Calanoida species along the reservoir.
Comparison of the distribution of the developmental stages of Copepoda at the three sites of the reservoir

As regards the distribution of the developmental stages of Calanoida and Cyclopoida at the three sites of the reservoir, it can be observed that Copepods produce a large number at the larval stage (nauplii) but significant mortality occurs before they reach the copepodit stage. The rate of the copepodit to the nauplii stage was four or five times less for Cyclopoida, whereas the rate for Calanoida was double or equal. This index is far more marked in the Cyclopoida, as can be seen in Table 5. 

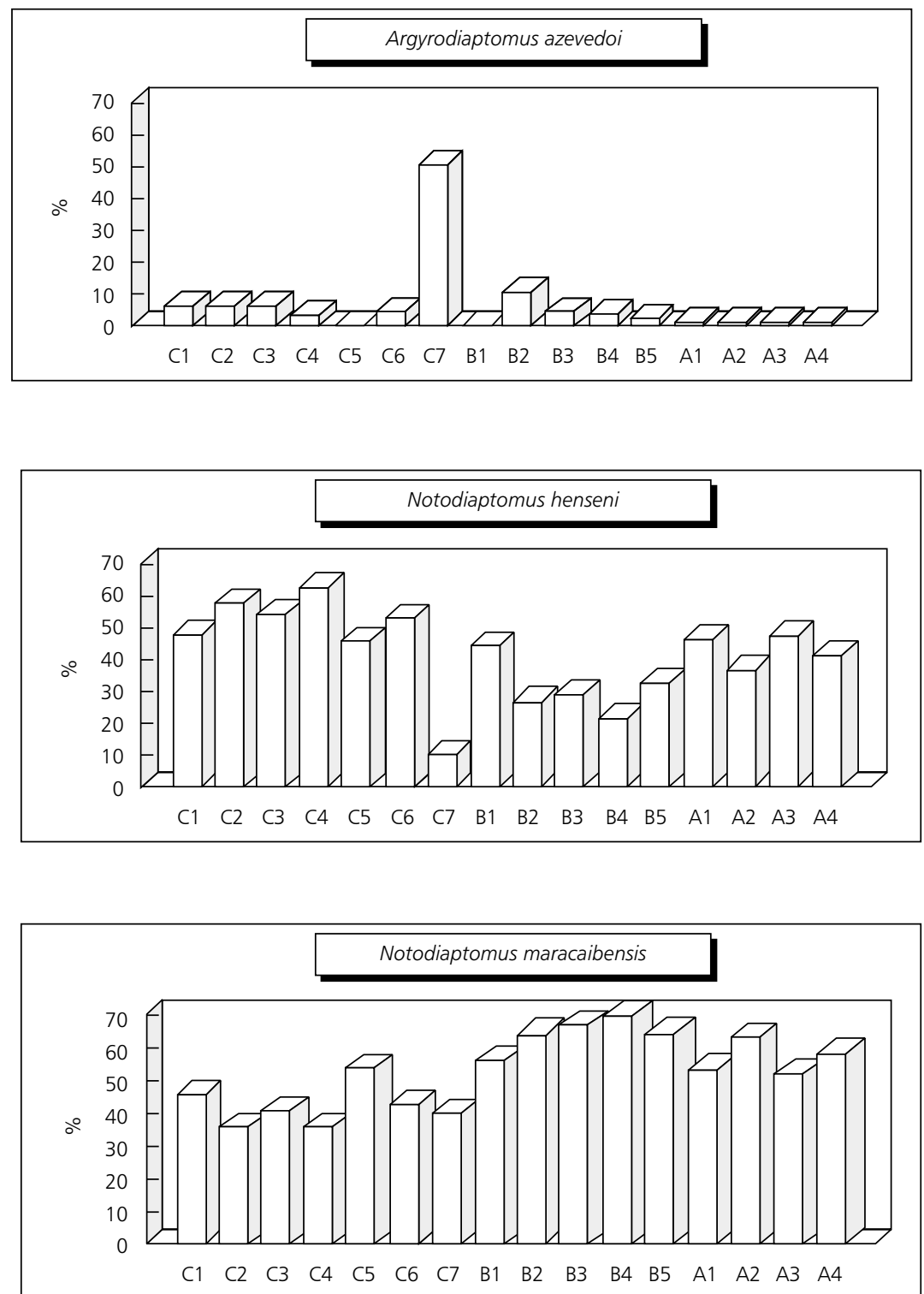

Fig. 6 - Relative abundance and horizontal distribution of Calanoida at the Caraipé (C), Araçagi (B) and Ararão (A) stations in the Tucuruí Reservoir.

If an analysis had been made of the relation between adults, copepodits and nauplii from the three sites, it would have been found that, in the case of Calanoida, the adults are more abundant at the Caraipé stations, there is a greater abundance of copepodits in Ararão, with nauplii predominating in Araçagi. A higher contribution of females with eggs sacs of Notodiaptomus maracaibensis was observed in Caraipé (19.7\% of the total Calanoida) than in other regions, and females with egg sacs of Notodiaptomus henseni and Argyrodiaptomus azevedoi abounded more at in the Araçagi stations (20.4\% and $61.5 \%$, respectively).

There was a greater abundance of the adult stage of Cyclopoida, represented mainly by Thermocyclops minutus, at the Araçagi and Ararão stations, while there were fewer nauplii and copepodits. 
TABLE 5

Percentage (\%) of adult, copepodit and naupliae phases of Calanoida and Cyclopoida found at the three sites of the Tucuruí Reservoir.

\begin{tabular}{|l|c|c|}
\hline & Calanoida & Cyclopoida \\
\hline ARARÃO & & 26.5 \\
\hline adult & 27.5 & 14.0 \\
\hline copepodit & 33.2 & 59.0 \\
\hline nauplii & 39.3 & \\
\hline ARAÇAGI & & 31.3 \\
\hline adult & 13.9 & 9.8 \\
\hline copepodit & 28.2 & 58.8 \\
\hline nauplii & 58.0 & \\
\hline CARAIPÉ & & 16.0 \\
\hline adult & 35.9 & 17.2 \\
\hline copepodit & 18.6 & 66.8 \\
\hline nauplii & 45.4 &
\end{tabular}

Females of Thermocyclops minutus with egg sacs were found in a higher proportion at the Ararão stations and those with Thermocyclops decipiens egg sacs were more plentiful at Caraipé. This evidence indicates that closely related species preferentially use different sites to reproduce, possibly to avoid interspecific competition.

\section{Diversity and similarity of the zooplankton species at the three sites}

Table 6 presents the indexes of similarity and diversity of species, calculated for Cladocera and Rotifera. The highest indexes of diversity were found at the Ararão and Araçagi stations, mainly for Rotifera, which presented greater equability at these stations (between 0.81 and 0.91 ), while less equability was found at the Caraipé stations (between 0.39 and 0.82), demonstrating a higher contribution from few species.

The indexes of similarity varied from 70 to 100 for Cladocera and from 21 to 44 for Rotifera, pointing to a more differentiated composition and distribution of the Rotifera species and a broader and more similar distribution of the Cladocera species in the Tucuruí Reservoir. In the cases of both Cladocera and Rotifera, the closest similarity was observed among the stations corresponding to the areas delimited as Caraipé ( $\mathrm{C} 1$ to $\mathrm{C} 7)$, Araçagi (B1 to B5) and Ararão (A1 to A4), while analyses showed less similarity occurring between the Caraipé and Araçagi stations and between the Araçagi and the Ararão stations.

TABLE 6

Maximum and minimum values found for the index of similarity (S), equitability (E) and species diversity $\left(\mathrm{H}^{\prime}=\right.$ bits/ind) at the Caraipé, Araçagi and Ararão stations.

\begin{tabular}{|c|c|c|c|c|}
\hline \multirow{2}{*}{ Stations } & \multicolumn{2}{|c|}{ Rotifera } & \multicolumn{2}{c|}{ Cladocera } \\
\cline { 2 - 5 } & \multicolumn{2}{|c|}{ Similarity: $21-44$} & Dimilarity: $90-100$ \\
\cline { 2 - 5 } & Diversity & Equitability & $1.99-2.50$ & $0.65-0.77$ \\
\hline Caraipé & $1.75-3.75$ & $0.39-0.83$ & $1.89-2.45$ & $0.55-0.71$ \\
\hline Ararão & $3.48-3.65$ & $0.79-0.91$ & $1.85-2.64$ & $0.62-0.92$ \\
\hline Araçagi & $3.12-3.52$ & $0.81-0.86$ & Equitability \\
\hline
\end{tabular}




\section{DISCUSSION}

According to Rocha et al. (1999), reservoirs are considered favorable environments for the development of zooplankton communities. The complex structure of reservoirs derives from the trophic state (related to the time of formation and artificial enrichment), the morphometry (related to shape and size) and operational regimen (retention time and outflow), which lead to the formation of different compartments within the same system.

From this standpoint, the physical, chemical and biological differences found at each station and study area characterize the different compartments that exist at the Tucuruí Reservoir, such as the Ararão, Araçagi and Caraipé. Each compartment has physical peculiarities (morphometry and areas previously deforested or not) that influence the water flow, the retention time of materials, the incorporation of nutrients, the production of organic matter, and the establishment of populations.

The water in the Ararão compartment, located in a more open area in the upper portion of the reservoir, stays there less time (greater flow). It is an area devoid of islands where the water circulates more freely, but it contains flooded forest with layers of anoxic water or reduced amounts of oxygen and a greater thermal homogeneity. The lower values of chlorophyll and zooplanktonic biomass indicate the establishment of smaller species with a faster reproductive cycle, such as the rotifers (Ascomorpha ecaudis and Polyarthra cf vulgaris) and small cladocerans (such as Bosmina hagmanni and Moina minuta). The Araçagi compartment corresponds to the area closest to the dam, with prior deforestation and deeper stations, higher values of oxygen in comparison to the Ararão, and a smaller zooplanktonic biomass, demonstrating the effect of low water retention time in the structuring of its populations. Both compartments, however, despite the smaller zooplanktonic biomass, presented a greater diversity due to the equability among species in comparison to the Caraipé.

The Caraipé compartment is shallower and has extensive areas not deforested before being flooded. The compartment contains several islands and its edges are markedly dendritical. These factors lead to longer retention times and, the- refore, to a greater material cycling among the system's biotic components, which contributes to increased organic production. The sampled stations in this area presented physical and chemical stratification, with low oxygen levels and anoxia at several stations. It was also noted that the larger species, such as Argyrodiaptomus azevedoi (Calanoida), Mesocylops longisetus (Cyclopoida), Trocosphaera aequatorialis (Rotifera) and $\mathrm{Da}$ phnia gessneri (Cladocera) were only present or were more abundant at the Caraipé stations, a fact that may be related to the greater availability of food as well as to the presence of larger refuge areas. In the case of the planktonic populations, some papers have demonstrated the influence of the presence of subsystems lateral to the principal axis of reservoirs (Kimmel et al., 1990; Betsil \& Van Den Avyle, 1994), a situation similar to the Caraipé region.

The compartmentalization of the Tucurui Reservoir, as it concerns the system's biotic components, is related to the reservoir's morphometry and the presence or absence of prior deforestation.

These factors contribute toward a reduction of the water flow and to the increase of areas that favour colonization by autotrophic organisms, such as periphytic and macrophytic aquatic algae, which forms differentiated niches for the establishment of other communities, contributing to species diversity and biomass production. The distribution and abundance of organisms and their relation to the presence of macrophytes have been mentioned by several authors (Gehrs, 1974; Rocha, 1978). Gehrs (1974), for instance, mentions the relation between the distribution of Diaptomus clavipes and the presence of the macrophyte Potamogeton foliosus in a small reservoir in the United States and under experimental conditions, while Rocha (1978) discusses the macrophytic regions in the Represa do Lobo (State of São Paulo), an area of greater reproduction for Copepoda Calanoida Argyrodiaptomus furcatus.

Within this diversity of the system's habitats, the establishment of different species is a consequence of the environmental factors that can, directly or indirectly, influence the reproduction and survival of organisms, as postulated by the Theory of the Environment (Andrewarthra \& Birch, 1984). Thus, reproductive strategies and adapta- 
tions of species to abiotic (such as $\mathrm{pH}$, temperature, dissolved oxygen, water flow, etc.) and biotic (predation and competition for food resources) environmental factors must be taken into account in distribution analyses (occurrence and density) of a system's species. Studies developed by several authors have shown the magnitude of environmental effects, principally temperature, food and predation, on the life cycle of species. This can be observed by the differences in average fecundity, development time of the different life cycles, longevity and size of organisms (Espíndola \& Niselli, 1996; Rietzler, 1991; Rietzler \& Espíndola, 1998).

One must also consider that areas with a greater horizontal water circulation, such as Ararão and Araçagi, can have a stronger effect on the structure of the zooplanktonic community, since the constant input of water leads to changes in the environmental conditions, as explained by Threlkeld $(1983,1986)$. Other factors, such as wind, whose influence is more effective in more open areas, may contribute toward the spatial heterogeneity of populations.

The findings of this study allow one to conclude that, despite the apparent homogeneity of the planktonic community, the organisms have a differentiated spatial distribution with a greater or lesser number of species, biomass or density, depending on environmental conditions, as also discussed by Riley (1976), Urabe \& Murano (1986), Betsil \& Van Den Avyle (1994), among others. Therefore, studies involving biological communities should comprehend the largest possible number of samplings in order to better characterize the structure of communities, as well as the other metabolic processes deriving from the workings of an ecosystem. Otherwise, according to Lind et al. (1993), samples taken from a single sampling station may be inadequate, to characterize spatially and temporarily heterogeneous and dynamic systems.

Acknowledgments - The authors wish to express their appreciation for the financial support of the Brazilian research funding institution, $\mathrm{CNPq}$, as well as for the infrastructure and logistical support provided by ELETRONORTE to carry out the collections and laboratory analyses.

\section{REFERENCES}

ANDREWARTHRA, M. C. \& BIRCH, L. C., 1984, The ecological web: more on the distribution and abundance of animals. The University of Chicago Press, Chicago.

ARMENGOL, J. \& SAAB, F., 1990, Annual and longitudinal changes in the environmental conditions in three consecutive reservoirs of Guadiana river (W.Spain). Arch. Hydrobiol. Beih., 33: 679-687.

BETSIL, R. K. \& VAN DEN AVYLE, M. J., 1994, Spatial heterogeneity of reservoir zooplankton: a matter of timing? Hydrobiologia, 277: 63-70.

BURGIS, M. J., 1969, A preliminary study of the ecology of zooplankton in Lake George, Uganda. Verh. Internat. Verein. Limnology, 17: 297-302.

CRYER, M. \& TOWNSEND, C. R., 1988, Spatial distribution of zooplankton in a shallow eutrophic lake, with a discussion of its relation to fish predation. Journal of Plankton Research, 10(3): 487-501.

EDMONDSON, W. T. \& WINBERG, G. G., 1971, A manual of methods for the assessmemnt of secondary productivity in freshwater. Blackwell Scientific Publications, Oxford, Handbook 17, IBP.

EDMONDSON, W. T., 1959, Freshwater Biology. John Wiley \& Sons Inc., New York, 1248p.

ESPÍNDOLA, E. L. G. \& NISELLI, R., 1996, Análise da dinâmica populacional de Notodiaptomus conifer, Sars, 1901 (Copepoda Calanoida): uma abordagem experimental. Acta Limnológica Brasiliensia, 8: 1-12.

ESTEVES, F. A., 1988, Fundamentos de Limnologia. Editora Interciência/FINEP, Rio de Janeiro, 575p.

GEHRS, C. W., 1974, Horizontal distribution and abundance of Diaptomus clavipes Schacht in relation to Potamogeton foliosus in a pond and under experimental conditions. Limnology and Oceanography, 19(1): 100-104.

GOLTERMAN, H. L., CLYMO, R. S. \& ONHSTAD, M. A. M., 1978, Methods for physical and chemical analysis of freshwater. $2^{\text {th }}$ ed. Blackwell Scientific Publication, Oxford, 213p.

HART, R. C., 1978, Horizontal distribution of the Copepod Pseudodiaptomus hessei in a subtropical Lake Sibaya. Freshwater Biology, 8(5): 415-422.

HAYWARD, R. S. \& VAN DEN AVYLE, M. J., 1986, The nature of zooplankton spatial heterogeneity in a nonriverine impoundment. Hydrobiologia, 131: 261271 .

HUTCHINSON, G. E., 1967, A treatise on limnology (Introduction to lake biology and the limnoloplankton). John Wiley \& Sons, Inc., New York, 2 vol., $1115 \mathrm{p}$. 
INFANTE, A. G., 1995, Vertical and horizontal distribution of the zooplankton in lake Valencia. Acta Limnologica Brasiliensia, 6: 97-105.

JONES, R. I., FULCHER, A. S., JAYAKODY, J., LAYBOURN-PARRY, J., SHINE, A. J., WALTON, M. C. \& YOUNG, J. M., 1995, The horizontal distribution of plankton in a deep, oligotrophic lake - Loch Nees, Scotland. Freshwater Biology, 33: 161-170.

KIMMEL, B. L., LIND, O. T. \& PAULSON, L. J., 1990, In: K. W. Thornton, B. L. Kimmel \& E. F. Payne (eds.), Reservoir limnology: ecological perspectives. A Wiley-Intersciewnce Publication. John Wiley \& Sons, Inc., 246p.

KOSTE, W., 1978a, Rotatorie die Radertiere Mitteuropas. I. Texband Gebruder Borntraeger, Stuttgart, 673p.

KOSTE, W., 1978b, Rotatoria. Die radertiere mitteuropas II. Textband. Gebruder Borntrauger, Berlim, Stuttgart, $237 \mathrm{p}$.

LIND, O. T., TERREL, T. T. \& KIMMEL, B. L., 1993, In: M. Straškraba, J. G. Tundisi \& A. Duncan (eds.), Comparative reservoir limnology and water quality management. Kluwer Academic Publishers. Dordrecht. The Netherlands, pp. 57-67.

MATSUMURA-TUNDISI, T., 1984, Occurence of species of the genus Daphnia in Brazil. Hydrobiologia, 112: 161-165.

MATSUMURA-TUNDISI, T., 1986, Latitudinal distribution of Calanoida Copepods in freshwaters aquatic systems of Brazil. Rev. Brasil. Biol., 46(3): 527-553.

MONTÚ, M. \& GOEDEN, I. M., 1986, Atlas dos Cladocera e Copepoda (Crustacea) do estuário da Lagoa dos Patos (Rio Grande, Brasil). Nerítica, 1(2): 1-34.

PENNAK, G. E., 1953, Freshwater invertebrates of the United States. The Ronald Press Company, New York, $769 \mathrm{p}$.

REID, J. W., 1985, Calanoid copepods (Diaptomidae) from Costal lakes, State of Rio de Janeiro, Brazil. Proc. Biol. Soc. Was., 98(3): 574-590.

RIETZLER, A. C. \& ESPÍNDOLA, E. L. G., 1998, Microcystis as a food source for copepods in a subtropical eutrophic reservoir. Verh. Internat. Verein. Limnol., 26: 2001-2005.

RIETZLER, A. C., 1991, Estudo da dinâmica de populações de Copepoda Calanoida na Represa do Lobo ("Broa"). Dissertação de Mestrado, EESC/USP, São Carlos, 196p.

RILEY, G. A., 1976, A model of plankton patchiness. Limnology and Oceanography, 21(6): 873-880.

ROCHA, O. \& MATSUMURA-TUNDISI, T., 1976, Atlas do zooplâncton da Represa do Lobo (Broa). UFSCar, São Carlos, Série Atlas, 1 vol., 68p.

ROCHA, O., 1978, Flutuação sazonal e distribuição da população de Diaptomus furcatus, Sars (Copepoda Calanoida) na Represa do Lobo ("Broa"), São Carlos, SP. Dissertação de Mestrado, USP, São Carlos, 147p.
ROCHA, O., MATSUMURA-TUNDISI, T., ESPÍNDOLA E. L. G., ROCHE, K. F. \& RIETZLER, A. C., 1999, Ecological theory applied reservoir zooplankton. In: J. G. Tundisi \& M. Straškraba (eds.), Theoretical reservoir ecology and its applications. São Carlos, São Paulo, 592p.

SHANNON, C. E. \& WEAVER, W., 1949, The mathematical theory of communication. Urbana, Univer: Illinois Press, 125p.

SMIRNOV, N. N. \& TIMMS, B. V., 1983, A revision of the Australian Cladocera (Crustacea). Record of the Australian Museum, Suppl 1, 132p.

SMIRNOV, N. N., 1974, Fauna of the USSR - Crustacea (Chydoridae). Jerusalém: Peter Publishing House Ltd, $644 \mathrm{p}$.

SORENSEN, T., 1948, A method of stablishing groups of equal amplitude in plant sociology based on similarity of species content and its application to analysis of vegetation on Danish commons. Biol. Skr, 5(4): 1-34.

SOTO, D., VILA, I. \& VILLALOBOS, B., 1984, Temporal and spatial distribution of Rotifera in a Chilean reservoir: a possible effect of impoundment hydrodynamics. Hydrobiologia, 114: 67-74.

STAVN, R. H., 1971, The horizontal-vertical distribution hypothesis: Langmuir circulations and Daphnia distribution. Limnology and Oceanography., 16(2): 453466.

THRELKELD, S. T., 1983, Spatial and temporal variation in the summer zooplankton community of a riverine reservoir. Hydrobiologia, 107: 249-254.

THRELKELD, S. T., 1986, Life table responses and population dynamics of four cladoceran zooplankton during a reservoir flood. Journal of Plankton Research, 8(4): 639-647.

TUNDISI, J. G., 1981, Typology of reservoirs in Souther Brazil. Verh. Internat. Verein. Limnology, 21: 10311039

TUNDISI, J. G., 1990, Distribuição espacial, seqüência temporal e ciclo sazonal do fitoplâncton em represas: fatores limitantes e controladores. Rev. Brasil. Biol., 50(4): 937-955.

URABE, J. \& MURANO, M., 1986, Seasonal and horizontal variations in the zooplankton community of Ogochi Reservoir, Tokyo. Bolm of Plankton Society of Japan, 33(2): 101-112.

WATSON, N. H. F. \& CARPENTER, G. F., 1974, Seasonal abundance of crustacean zooplankton and net plankton biomass of lakes Heron, Erie and Ontario. J. Fish. Res. Board of Canada, 31(3): 309-317. 